Board \#73 ?

Integrative Exercise Physiology

Influence Of Compression Garments On Physiological And Perceptual Responses During Intermittent Anaerobic Running And Recovery

Anthony S. Leicht, Melissa J. Crowe, Wade H. Sinclair, Rebecca M. Sealey. James Cook University, Townsville, Australia.

Email: Anthony.Leicht@jcu.edu.au

PURPOSE: Compression garments have gained recent prominence and promoted to be beneficial during exercise performance and recovery. The aim of the current study was to examine the influence of compression garments on cardiovascular, thermoregulatory, metabolic and perceptual responses during intermittent anaerobic running and subsequent recovery. METHODS: Fifteen young, healthy adults volunteered for this study and provided written informed consent in line with institutional ethics approval. Participants completed three intermittent maximal anaerobic running power (MARP) tests while wearing, in a randomised order, no compression garments (CONTROL), a lower body compression garment (TIGHTS) or combined upper and lower body compression garments (SHIRT/TIGHTS). Heart rate (HR), core temperature (TC), lactate (La) and rating of perceived exertion (RPE) were monitored during the MARP tests and the subsequent active (10 mins) and passive (20 mins) recovery phases. Significant $(p<0.05)$ differences over time and between garment conditions were determined by repeated measures ANOVA and Tukey's HSD post-hoc tests. RESULTS: During the MARP test, HR increased similarly for each garment condition with maximal values of $H R, T C, L a, R P E$ and performance not significantly different between garment conditions. However, TC overall was significantiy greater for SHIRT/TIGHTS compared with TIGHTS during the MARP while greater RPE was exhibited for TIGHTS compared to CONTROL during the early stages of the MARP. During recovery, HR, RPE and La decreased similarly for each garment condition. CONCLUSIONS: Compression garments significantiy altered thermoregulatory and perceptual responses without significant changes in anaerobic performance or recovery responses following intermittent anaerobic running.

\section{Board \#74}

\section{Integrative Exercise Physiology}

\section{Influences Of Experimental Dyslipidemia On Murine} Diabetic Neuropathy

Brianne L. Guilford, Janelle M. Ryals, Douglas E. Wright. University of Kansas Medical Center, Kansas City, KS.

Diabetic neuropathy (DN) is a debilitating complication of diabetes, affecting over $50 \%$ of diabetic patients. Dyslipidemia, oxidative stress, and mitochondrial dysfunction may be important etiological factors in the pathogenesis of DN, but the contributions of these variables in DN are controversial. Some of the controversy may stem from the fact that neuropathy in rodents is subtie, thus making it difficult to identify important modulators that contribute to the development and maintenance of neuropathy. PURPOSE: We hypothesize that mitochondrial dysfunction is a key mechanism involved in DN, but may be more apparent in settings of dyslipidemia and oxidative stress. The ultimate goal of these studies is to examine the effects of a high-fat diet on the progression of DN to understand how diet-induced oxidative stress influences mitochondrial function in peripheral sensory neurons. METHODS: Comparisons of neuropathy indices and serum lipids were made between streptozotocin-induced diabetic and nondiabetic C57Bl/6 mice consuming a standard or high-fat diet for 9 weeks. RESULTS: In nondiabetic mice, the high-fat diet significantly decreased proprioception and increased motor nerve conduction velocity, but mechanical sensitivity, thermal sensitivity, intraepidermal nerve fiber density (IENFD), and sensory nerve conduction velocities were not different from nondiabetic mice fed a standard diet. In diabetic mice, addition of the high-fat diet significantly increased mechanical sensitivity (allodynia) but did not alter thermal sensitivity, proprioception, IENFD, or sensory or motor nerve conduction velocities. The high-fat diet increased serum total cholesterol and LDL cholesterol (LDL-C) but did not change serum triglycerides in nondiabetic mice. Diabetes elevated total cholesterol but did not affect LDL-C or triglycerides in mice fed a standard diet. The combination of diabetes and the high-fat diet increased triglycerides but total cholesterol and LDL-C were not different compared to diabetics fed a standard diet. CONCLUSIONS: In conclusion, a high-fat diet alters sensory-related behavior and nerve conduction velocity. Introduction of a high-fat diet in addition to diabetes switched sensory-related behavior from moderate mechanical insensitivity to mechanical allodynia. Thus, rather than exacerbating $\mathrm{DN}$, it appears a high-fat diet may switch the neuropathy phenotype from insensate to painful in this mouse strain. Current studies are addressing mechanistic differences in these neuropathic phenotypes as we examine mitochondrial dysfunction, oxidative stress, and exercise interventions that improve DN in this dyslipidemic model.

\section{Board \#75}

\section{Integrative Exercise Physiology}

\section{Moderate Caloric Restriction Suppresses microRNAs miR-696 and miR-199a in a Voluntary Running Wheel Model}

R. Andrew Shaneiy1, Amy M. Knab1, Jenna M. Dixon2, Meghan Kusper3, L. Grant Canipe III3. 'Appalachian State University, Kannapolis, NC. ${ }^{2}$ lowa State University, Ames, IA. ${ }^{3}$ Appalachian State University, Boone, NC. Email: shanelyra@appstate.edu

The transcriptional coactivator PGC-1a is central to skeletal muscle adaptation to increased physical activity. SIRT1, an energy sensing deacetylase, and important regulator of transcription, plays a role in the benefits of caloric restriction. SIRT1 is also a regulator of PGC-1a in skeletal muscle. MicroRNAs (miRNAs) are short noncoding RNA molecules involved in post-transcriptional regulation of gene expression by binding to target mRNA. Expression levels of miRNAs can be altered by physiological stimuli. miR-696 and miR-199a are reported to target PGC-1a and SIRT1, respectively. Neither expression of miR-696 nor miR-199a has been studied in the adaptation of skeletal muscle to physical activity and moderate caloric restriction (CR). PURPOSE: We tested the hypothesis that moderate CR in physically active mice would suppress expression of miR-696 and miR1.99a. METHODS: Male C57BL/6J mice ( $9 \mathrm{wks}$ old) were individually housed and assigned to one of two groups: ad libitum feeding (AL, $n=7)$ or moderate CR ( $n=6)$. The AL mice had free access to food and a running wheel for 23 days. The moderate CR mice had free access to a running wheel for 23 days and free access to food for the initial 8 days, and then moderate CR for 15 days. The moderate CR protocol was implemented to achieve a $20 \%$ reduction in body mass within 3 days. Body mass reduction was then maintained for the remainder of the experiment. Running distance was measured daily. Total RNA was isolated from the plantaris (PLA). Total RNA and microRNA were reverse transcribed in separate reactions to measure expression levels of COXI, PGC-1a, and SIRT1, or miR-696, miR-199a. The relative copy number of mitochondrial (mt) DNA was measured in total DNA (nuclear and mitochondrial) extracted from PLA. RESULTS: The moderate $\mathrm{CR}$ group required a $26 \%$ decrease in food intake to achieve the $20 \%$ decrease in body mass. During the 15 days of moderate CR mice ran $10.5 \mathrm{~km} /$ day while the $A L$ mice ran $6.9 \mathrm{~km} /$ day $(p<0.05)$. $m$ tDNA copy number and COXI expression were significantly $(p<0.05)$ greater, $27 \%$ and $64 \%$ respectively, in moderate CR mice, while PGC1 a expression was not affected ( $p>0.05$ ). However, miR-696 expression was suppressed $(70 \%, p<0.05)$ in moderate CR mice. Moderate CR resulted in increased SIRT1 expression $(66 \%, p<0.05)$ and conversely, decreased miR-199a expression $(58 \%, p<0.05)$. CONCLUSION: Compared to wheel running alone, moderate CR significantly increased running wheel distance, COX1 and SIRT1 mRNA expression, mtDNA copy number, and suppresses microRNAs miR-696 and miR-199a in physically active mice. These data suggest that moderate CR may regulate mitochondrial biogenesis through posttranscriptional mechanisms. 